\title{
A concise account of techniques available for shipboard sea state estimation
}

\author{
Nielsen, Ulrik Dam
}

Published in:

Ocean Engineering

Link to article, DOI:

10.1016/j.oceaneng.2016.11.035

Publication date:

2017

Document Version

Peer reviewed version

Link back to DTU Orbit

Citation (APA):

Nielsen, U. D. (2017). A concise account of techniques available for shipboard sea state estimation. Ocean Engineering, 129, 352-362. https://doi.org/10.1016/j.oceaneng.2016.11.035

\section{General rights}

Copyright and moral rights for the publications made accessible in the public portal are retained by the authors and/or other copyright owners and it is a condition of accessing publications that users recognise and abide by the legal requirements associated with these rights.

- Users may download and print one copy of any publication from the public portal for the purpose of private study or research.

- You may not further distribute the material or use it for any profit-making activity or commercial gain

- You may freely distribute the URL identifying the publication in the public portal

If you believe that this document breaches copyright please contact us providing details, and we will remove access to the work immediately and investigate your claim. 


\title{
A Concise Account of Techniques Available for Shipboard Sea State Estimation
}

\author{
Ulrik Dam Nielsen ${ }^{1,2 *}$ \\ ${ }^{1}$ DTU Mechanical Engineering, Technical University of Denmark, DK-2800 Kgs. Lyngby, Denmark \\ ${ }^{2}$ Centre for Autonomous Marine Operations and Systems, AMOS-NTNU, NO-7491 Trondheim, Norway
}

\begin{abstract}
This article gives a review of techniques applied to make sea state estimation on the basis of measured responses on a ship. The general concept of the procedures is similar to that of a classical wave buoy, which exploits a linear assumption between waves and the associated motions. In the frequency domain, this assumption yields the mathematical relation between the measured motion spectra and the directional wave spectrum. The analogy between a buoy and a ship is clear, and the author has worked on this wave buoy analogy for about fifteen years. In the article, available techniques for shipboard sea state estimation are addressed, but with a focus on only the wave buoy analogy. Most of the existing work is based on methods established in the frequency domain but, to counteract disadvantages of the frequency-domain procedures, newer studies are working also on procedures formulated directly in the time domain. Sample results from several studies are included, and the main findings from these are mentioned.
\end{abstract}

Key words: Sea state estimation; Wave buoy analogy; Vessel responses; Frequency domain; Time domain.

\section{INTRODUCTION}

In today's maritime world, the operation of ships requires careful monitoring of the related costs while, at the same time, ensuring a high level of safety. Shipboard decision support systems may enable a ship's crew to reduce costs and minimise risks while sailing, so that the performance is optimised. A ship's performance with respect to safety and fuel efficiency is in general negatively influenced by the encountered waves. Consequently, it is of particular importance to estimate the surrounding sea state, and any shipboard decision support system needs to have information about the encountered waves as input for the system to be the most accurate and reliable.

*Email: udn@mek.dtu.dk 
Trustful means for sea state estimation include floating wave buoys, which are primary tools used to collect statistical ocean wave data. However, wave buoys are not practical for a sailing ship requiring (precise) sea state information in real-time and at its actual geographical position. On the other hand, the analogy between a ship and a floating buoy naturally suggests to using the ship itself as a kind of wave buoy. Thus, a number of research studies have explored this 'wave buoy analogy' in the past, and the author of the present paper has worked extensively on the topic for about the last fifteen years.

This paper presents a concise account of techniques for shipboard sea state estimation using measured vessel responses, resembling the concept of a traditional wave buoy. Moreover, newly developed ideas for shipboard sea state estimation are introduced. The account, or review, is not necessarily complete, as it primarily reflects the author's personal experience and background; obtained alone and together with national as well as international colleagues. However, it is believed that the author has come across most of the work carried out within the particular field, so other fundamental studies, not related to the present author, will also be cited; without the ambition to list every single reference from the literature.

Although other means for shipboard sea state estimation exist, based on, e.g., the use of X-band navigational radars or over-the-bow looking devices, those means will not be mentioned herein and, hence, shipboard sea state estimation refers in the following to only the wave buoy analogy, where sea state estimation is conducted on the basis of measured vessel responses. Onwards, sea state estimation will at most places be shortened by SSE.

1.1. Past Work and Literature. Until the 1970'ies, little work on shipboard SSE had been done, but early researches (e.g. Lindemann and Nordenstrøm, 1975; Lindemann et al., 1977; Robinson, 1990; Debord and Hennessy, 1990; Francescutto, 1993) on in-service monitoring systems combined with decision support tools emphasised the need for estimates of the on-site sea state; at the actual position of the advancing vessel. Some of the initial studies on shipboard SSE (Takekuma and Takahashi, 1973; Pinkster, 1978) did not consider ships with forward speed, and although attempts were made to introduce forward speed in shipboard SSE, notably by Japanese studies (Isobe et al., 1984; Kobune and Hashimoto, 1986; Hirayama, 1987; Iseki et al., 1992; Saito et al., 2000; Maeda et al., 2001), the first study to strictly consider the Doppler shift, implying a 1-to-3 relationship between encounter frequency and wave frequency for certain conditions in following sea, was made by Iseki and Ohtsu (2000). Since then several studies with good results have been published for ships with forward speed (Iseki and Terada, 2002; Iseki, 2004; Nielsen, 2006; Nielsen and Stredulinksy, 2012; Nielsen and Iseki, 2012; Nielsen et al., 2013; Montazeri et al., 2016a; Montazeri, 2016); all considering full-scale data of different vessels. A number of studies have also been made in relation to station keeping and dynamic positioning, where shipboard SSE has been made with success for 
ships without forward speed (Waals et al., 2002; Tannuri et al., 2003; Pascoal et al., 2007; Simos et al., 2007; Sparano et al., 2008; Pascoal and Soares, 2009).

1.2. Content and Composition of Paper. Different mathematical models exist for the wave buoy analogy, and the main principles will be outlined in Section 2. It is shown that shipboard SSE can be carried out either in the frequency domain or in the time domain, and, based on the setting, Sections 3 and 4 provide summaries of the fundamental assumptions and the different mathematical models which are applied depending on the particular domain, being it time or frequency. Sample results taken from several of the author's previous application studies, relating to both frequency and time domain calculations, are included in Section 5. Finally, concluding remarks are given in Section 6.

\section{WAVE BUOY ANALOGY}

Most of today's marine vessels are instrumented with sensors to record, e.g., global motion components such as heave, pitch, and vertical acceleration at specific position(s) relative to the centre of gravity. In this sense, vessels resemble classical wave buoys; although the latter typically have much simpler geometrical forms compared to the hull of a ship. Anyhow, the response recordings from marine vessels can be processed to facilitate estimation of the on-site sea state, making the analogy to floating wave buoys by relating the measurements and the sea state through a mathematical model, see Figure 1.

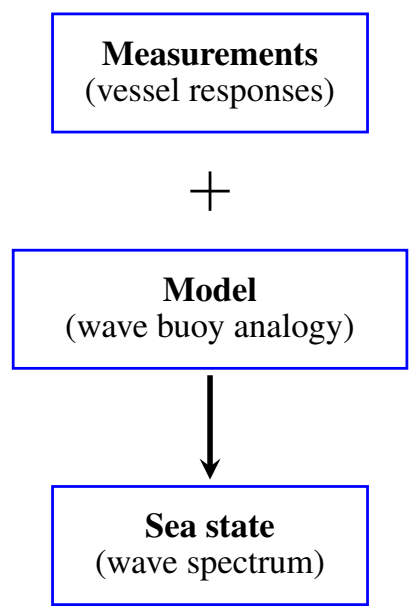

FIGURE 1. Combination of wave-induced response measurements and a mathematical model can be used to deduce information about the on-site sea state. (Nielsen et al., 2016)

2.1. Main Assumption. In mild and moderate wave climate, the wave-induced six degrees-of-freedom motion of a ship and associated structural loads are often assumed to be linear with the incident waves, 

(Nielsen, 2006)

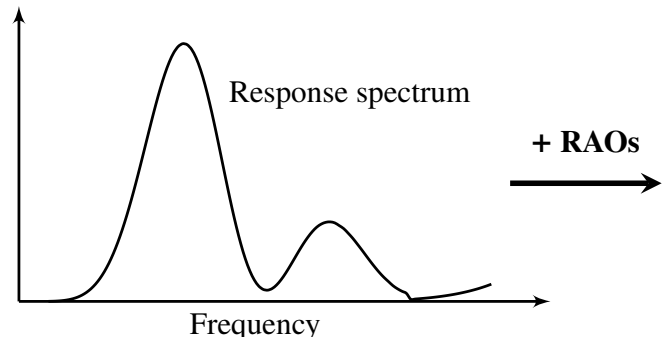

Frequency degree of approximation, and often they provide good results. conditions, RAOs can be still used to calculate responses of ships.

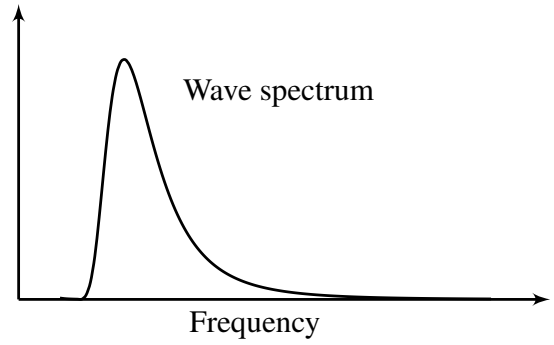

Frequency

FIGURE 2. Main principle of the wave buoy analogy when it is formulated in the frequency domain. (Nielsen et al., 2016)

meaning that the amplitudes of those responses are proportional to the wave amplitudes in regular waves. Consequently, the responses can be quantified in irregular waves by adding together results from regular waves with different amplitudes, wavelengths and propagation directions.

The linear assumption between waves and associated responses facilitates the use of transfer functions, or response amplitude operators (RAOs), that express how waves are transferred into responses. State-of-theart techniques for calculation of RAOs include 3-dimensional panel codes considering potential wave theory; sometimes supplemented with CFD based on the full set of Navier-Stoke's equations and/or considering other nonlinear effects. Nonetheless, strip theory calculations are still widely used, due to their adequate

In theory, RAOs are not necessarily accurate in severe waves, where a nonlinear relationship between waves and responses would/could occur. In practice, however, many studies have shown that even in severer wave

2.2. Frequency and Time Domain Approaches. The majority of previous work on the wave buoy analogy (e.g. Hua and Palmquist, 1994; Iseki and Ohtsu, 2000; Tannuri et al., 2003; Nielsen, 2006, 2008b; Pascoal et al., 2007; Montazeri et al., 2016a) is based on a solution formulated entirely in the frequency domain. This is illustrated in Figure 2, where a response spectrum is combined with RAOs, using spectral analysis, so that an estimate of the sea state is given in terms of a wave (energy) spectrum. Studies have shown that, in practice, wave estimation is improved by (optimally) selecting a set of three simultaneous vessel responses

Instead of a solution formulated in the frequency domain, derived by use of spectral analysis and with possible disadvantages, it has been suggested by Nielsen et al. $(2015,2016)$ to make the fitting of the measured response and the corresponding theoretically calculated one directly in the time domain (Fig. 3). In this 

former works (Nielsen et al., 2015, 2016) as will be outlined in Section 4.
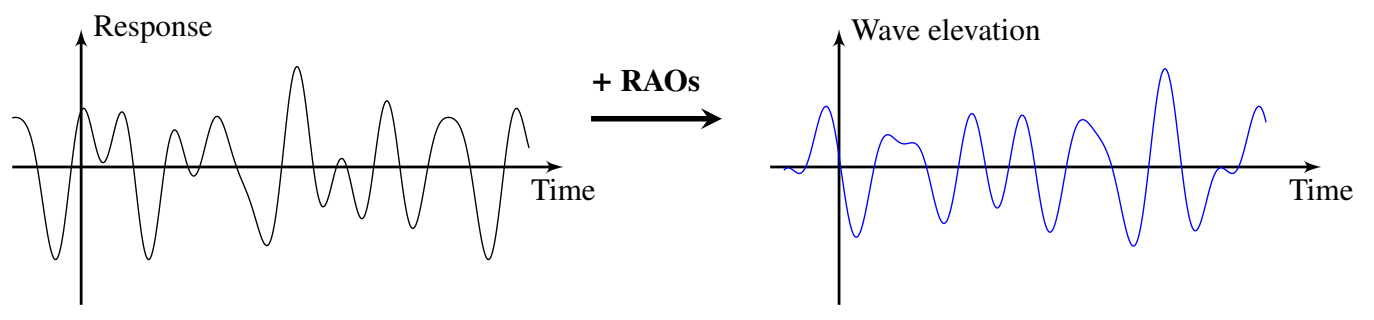

FIGURE 3. Conceptually, the wave buoy analogy can be formulated directly in the time

domain to give the actual wave elevation at the site of the vessel. (Nielsen et al., 2016)

sense, the approach is similar to a previous work by Pascoal and Soares (2009) that also formulate the governing equation directly in the time domain. However, the latter method (Pascoal and Soares, 2009), based on Kalman filtering, relies completely on availability of accurate RAOs, which is the main difference to the

In the next two sections, 3 and 4, the fundamentals of the techniques used for, respectively, frequency domain and time domain shipboard SSE are briefly described. As such, the sections can be read separately and have to some extent been formulated as stand-alone sections, which means that repetitions of fundamental assumptions and background occur.

\section{FREQUENCY DOMAIN APPROACHES FOR SSE}

Shipboard SSE is often considered in the frequency domain. Strictly speaking, the linear assumption about waves and associated responses needs, in this case, to be supplemented with additional assumptions: Firstly, the ocean waves and associated responses represent ergodic random processes (e.g. Ochi, 1990), so that stationarity, in a stochastic sense, applies within a certain period of response records at each estimation sequence. Secondly, the speed and the course of the ship (relative to waves) are constant in that period. Thus, response measurements from the particular period can be processed by spectral analysis using standard Fast Fourier Transformation (FFT) or multivariate autoregressive procedures (Nielsen, 2005, 2006), where the latter may sometimes be chosen because of the ability to automatically select the appropriate amount of smoothing; based on an order selection criterion. Traditionally, the mathematical model of shipboard SSE has then been formulated in terms of a comparison between the measured and the theoretically calculated spectral energy distribution of the considered responses. 
3.1. Comparison of Spectral Energy Distribution. A set of ship responses is considered, and the complexvalued transfer functions, $H_{i}\left(\omega_{e}, \chi\right)$ and $H_{j}\left(\omega_{e}, \chi\right)$ for the $\mathrm{i}$-th and $\mathrm{j}$-th responses, yield the theoretical relationship between the i-th and the j-th components of the response spectra $S_{i j}\left(\omega_{e}\right)$ and the directional wave spectrum $E\left(\omega_{e}, \chi\right)$ through the following integral equation

$$
S_{i j}\left(\omega_{e}\right)=\int_{-\pi}^{\pi} H_{i}\left(\omega_{e}, \chi\right) \overline{H_{j}\left(\omega_{e}, \chi\right)} E\left(\omega_{e}, \chi\right) d \chi
$$

where $\omega_{e}$ and $\chi$ are the encounter wave frequency and the relative wave heading, respectively, and the bar denotes the complex conjugate. The theoretical relationship expressed by Eq. (1) can be directly compared to a set of corresponding measured response spectra. Thus, the comparison constitutes the governing equation of the estimation problem that can be solved mathematically as a minimisation. It is vital to note that the wave spectrum is advantageously estimated in the wave frequency $(\omega)$ domain. This means that the speedof-advance - the so-called triple-valued function - problem in following sea needs to be considered. This problem, governed by the Doppler Shift, has been properly incorporated by Iseki and Ohtsu (2000), and in doing so, the mathematical relation between encountered (wave) frequency and true frequency is secured;

$$
\omega_{e}=\omega-\omega^{2} A, \quad A=\frac{U}{g} \cos \chi
$$

where $U$ is the forward speed of the ship, and $g$ is the acceleration of gravity. The triple-valued function problem exists when $\omega_{e}<\frac{1}{4 A}$ since, in this case, three wave frequencies correspond to one (positive) encounter frequency.

It should be understood that the left-hand side of Eq. (1) is estimated by measured data while the right-hand side is obtained through theoretical calculations. Consequently, a minimisation problem can be formulated and, casting the expressions into matrix notation, the objective is to minimise

$$
\chi^{2}(\mathbf{x}) \equiv\|\mathbf{A f}(\mathbf{x})-\mathbf{b}\|^{2}
$$

where $\|\cdot\|$ represents the $L_{2}$ norm. In the equation, the vector function $\mathbf{f}(\mathbf{x})$ expresses the wave spectrum $E(\omega, \chi)$ and the vector $\mathbf{b}$ contains the elements of the measured response spectra $S_{i j}\left(\omega_{e}\right)$, while the coefficient matrix A basically has its elements derived from the complex-valued transfer functions. Details are given by Nielsen (2006).

The minimisation problem given by Eq. (3) can be handled by different approaches. Two approaches that have received particular interest are formed by Bayesian modelling and parametric modelling. Bayesian modelling relies on finding the spectral components of a (discrete) frequency-directional wave spectrum, whereas parametric modelling assumes the directional wave spectrum to be formed by a set of parameterised wave spectra, e.g., JONSWAP using directional spreading parameters. Reports about the two procedures 
have been given in many studies (Iseki and Ohtsu, 2000; Iseki and Terada, 2002; Nielsen, 2006, 2008b; Pascoal and Soares, 2008; Tannuri et al., 2003; Pascoal et al., 2007; Simos et al., 2007), considering both simulated data and full-scale measurements with and without forward speed; and comparisons between the two modelling procedures have also been made. However, as pointed out by Nielsen and Stredulinksy (2012) and Nielsen et al. (2013), the two approaches should not been seen as competitors but rather as complementary, since each procedure has its own advantages and disadvantages.

3.2. Energy Equivalence: Comparison of Spectral Moments. In a recent PhD study, Montazeri (2016) suggests to formulate the governing equation from an energy conservation point-of-view, since the integrated variant of Eq. (1) is considered. Thus, the mathematical model is based on an equivalence of the spectral moments calculated by integrating the two sides of Eq. (1) with respect to frequency. Again, a set of responses is considered simultaneously using cross-coupling terms so that the governing equations read

$$
\int_{\omega_{e, l}}^{\omega_{e, h}} S_{i j}\left(\omega_{e}\right) d \omega_{e}=\int_{\omega_{l}}^{\omega_{h}} \int_{-\pi}^{\pi} H_{i}(\omega, \chi) \overline{H_{j}(\omega, \chi)} E(\omega, \chi) d \chi d \omega
$$

where indices $l$ and $h$ correspond to lower and higher frequency limits, respectively. The actual values of these limits are determined through a partitioning technique (Montazeri, 2016) introduced to separately estimate wind sea and swell components of the wave system, see Figure 4. The details of this technique are given by Montazeri et al. (2016a) and Montazeri (2016), but it is noteworthy that the ocean wave system is expressed through the sum of two parameterised wave spectra; one for swell and one for wind sea, and each taking the form $S_{\text {wave }}(\omega)$ of a general unidirectional spectrum for developing seas (Boukhanovsky and Soares, 2009):

$$
S_{\text {wave }}(\omega)=\alpha g^{2} \omega^{-r} \exp \left(-\beta \omega^{-n}\right) \gamma^{\exp \left[\frac{-\left(\frac{\omega}{\omega_{p}}-1\right)^{2}}{2 \sigma^{2}}\right]}
$$

where the fitting parameters are $\left[\alpha, \beta, \gamma, \sigma, \omega_{p}, r, n\right]$. A directional spectrum is obtained as

$$
E(\omega, \theta)=S_{\text {wave }}(\omega) D(\theta \mid \omega)
$$

with $D(\ldots)$ being a spreading function for wave direction $\theta$; satisfying the normality condition $\int_{-\pi}^{\pi} D(\theta \mid \omega) d \theta=1$. In its physical understanding, the equal sign in Eq. (4) should be read like "nearly equal to", so that the values of the fitting parameters, including the spreading function, are optimised by minimising the difference between the left- and right-hand sides of Eq. (4), with the wave spectrum $E(\omega, \chi)$ specified by Eq. (6)*; leaving all details to Montazeri et al. (2016a) and Montazeri (2016).

For the set of governing equations (Eq. 4) it is important to note that the right-hand side is explicitly written with account to the true (wave) frequency; and not the encounter frequency as is the case of the left-hand side

\footnotetext{
${ }^{*}$ Wave direction $\theta$ will be directly related to relative wave heading $\chi$ taking ship course into account.
} 


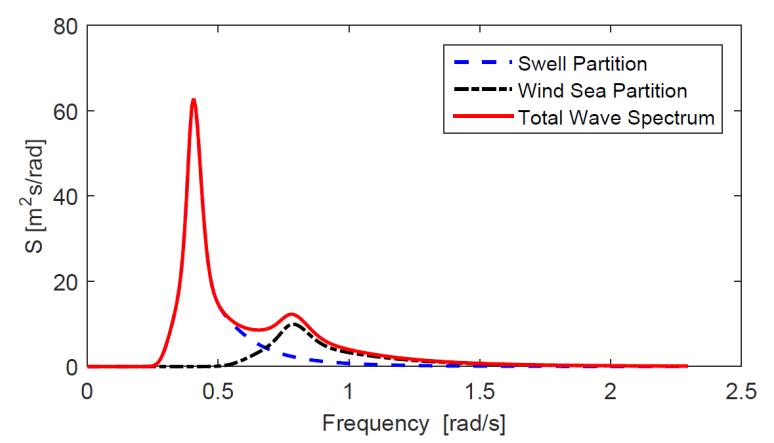

FIGURE 4. Spectral partitioning of wave spectrum into swell and wind sea components.

(Montazeri, 2016)

that represents the measurements. This particular formulation, with no need to transform between the two frequency domains for the theoretical calculation (i.e., the right-hand side), is possible since the energy must be the same in the two domains. Consequently, the exclusive consideration of equations based on energy conservation has the (positive) effect that the 1-to-3 relationship between encounter and wave frequency in following sea has not to be considered.

Compared to the other frequency-domain principle, outlined in subsection 3.1, the ambition with the 'energyequivalence principle', as stated by the authors (Montazeri, Nielsen and Jensen, 2016), is partly to make a practical robust procedure and partly to increase the computational efficiency of the wave buoy analogy; obviously, not compromising the ability to provide accurate sea state estimates.

3.3. Disadvantages of Frequency Domain Approaches. The outcome of the wave buoy analogy, when formulated in the frequency domain, consists of the on-site wave system's complete energy distribution, with frequency and directional information, and thus the approach is applicable to general decision support systems for safe and efficient marine operations. As reported in the literature, reasonable estimates of the wave spectrum can be expected (Nielsen, 2006, 2008b; Nielsen and Stredulinksy, 2012; Nielsen et al., 2013; Montazeri et al., 2016a), but the accuracy of the estimated sea state depends inherently on reliable transfer functions. Furthermore, the accuracy is highly dependent on the required spectral (response) analysis; hence, stationarity, or not, of the measurements may potentially influence the outcome (Møgster, 2015; Iseki and Nielsen, 2015). In principle, stationary operational conditions are necessary because a minimum time window, in the order 10-15 minutes, is needed to perform the spectral analysis. The reason is that if conditions are not stationary during the considered period, either because of a changing sea state or, more likely, as a result of speed and/or heading changes of the vessel, the sea state estimates are likely to be unreliable. Moreover, the need for a certain minimum time period has another consequence, as it implies that estimates, 
strictly speaking, are not real-time but will be backdated; which in turn may negatively influence response predictions made ahead of measurements, as discussed by Nielsen and Iseki (2015).

Altogether, these disadvantages of the frequency domain approaches have initiated studies where the solution, i.e. sea state estimate, is sought for directly in the time domain; to better accommodate partly nonstationary conditions. Moreover, work exists, in the time domain, to estimate the peak period of the sea state, with no other input than the response signal itself (Belleter et al., 2015; Brodtkorb et al., 2015). In the following, one of the time domain approaches looks at coupling this entirely "signal-based" estimation concept with the use of RAOs in a stepwise procedure to partly mitigate the fact that RAOs are always imperfect to some degree.

\section{TIME DOMAIN APPROACHES FOR SSE}

The central point of the procedures is a formulation of the estimation problem directly in the time domain, where focus is on real-time sea state updates obtained from continuous response measurements, with no need to consider a past measurement period.

Until now, only few works consider approaches for shipboard SSE directly in the time domain and, so far, the procedures, like those formulated in the frequency domain, rely on availability of accurate RAOs. Indeed, this is so for an elaborate procedure, building on a framework established by Kalman filtering, which will be addressed subsequently. However, as will be noted further below, it is possible to make a stepwise estimation procedure which couples an entirely signal-based procedure, estimating the peak wave period, with a model-based procedure, estimating wave height and phase. Herein, the signal-based step has no need for RAOs, whereas the model-based step makes use of RAOs. In the following, the two conceptually different approaches, based on, respectively, Kalman filtering and the stepwise procedure, will be concisely accounted for.

4.1. Wave Estimation Based on Kalman Filtering. An interesting study has been presented by Pascoal and Soares (2009) that propose a (high-speed) estimation algorithm established in a framework governed by Kalman filtering. Herein, the waves in-phase and quadrature components are introduced as the state variables, which means that (intrinsic) information about the actual wave elevation process is included in the solution. The mathematical details of the procedure are given by Pascoal and Soares (2009), so the following contains just a brief summary of the approach. ${ }^{\dagger}$

\footnotetext{
${ }^{\dagger}$ The present author has started recently to also work on this concept.
} 
The in-phase and quadrature components of a regular wave from (discrete) direction $\theta_{n}$, at discrete time $k$, constitute a set of state variables $X_{k}=\left[x_{1}, x_{2}\right]_{n}^{T}$. Relying on the electric filter analogy (St.Denis and Pierson, 1953), an irregular wave would be the sum of a (large) number of regular waves, implying that the associated sea state can be given in terms of an equivalent number of sets of state variables. In normal conditions, a sea state will be just very slowly varying and the hypothesis is that the state (in a Kalmancontext) at a next discrete time is as the present, except possibly for some variation due to the state noise $\xi_{k}$. Thus, the state equation reads

$$
X_{k+1}=X_{k}+\xi_{k}
$$

The state equation is supplemented with a measurement equation and, as the wave estimation is based on sensor recordings $r$ of $m$ available wave-induced responses, this equation is given by

$$
r_{m k}=C_{m k} X_{k}+\psi_{m k}
$$

where $C_{m k}$ is the measurement matrix transferring the states into a response (output) that is to be compared with a corresponding one available from measurement, while $\psi_{m k}$ is the measurement noise because of a sensor's limited capability. The equation is basically the equivalent of Eq. (1), but formulated in the time domain, and Eq. (8) represents, analogously, a linear relationship between a single harmonic wave component and an associated response component. As a consequence, the measurement matrix $C_{m k}$ can therefore be composed by the available (complex-valued) transfer functions $H$. The specific composition of the matrix is realised by expressing the measurement equation in its physical understanding, writing the irregular response $r$ as the sum of $n_{f}$ harmonic components, each represented in a total of $n_{\theta}$ wave directions:

$$
r=\operatorname{Re}\left(\sum_{j=1}^{n_{f}} \sum_{i=1}^{n_{\theta}} H_{j i} \times\left(X_{2 j-1, i}+\sqrt{-1} X_{2 j, i}\right) \times\left(\cos \left(\omega_{j} t\right)+\sqrt{-1} \sin \left(\omega_{j} t\right)\right)\right)
$$

From the equivalence between Eq. (8) and Eq. (9) it can be seen how the elements of the measurement matrix should be assigned. Part of the matrix is shown below for the $\mathrm{j}$-th frequency, $\mathrm{i}$-th direction, $\mathrm{m}$-th response and k-th time instant, respectively

$$
\begin{aligned}
C_{j i m k}= & {\left[\operatorname{Re}\left[H_{j i m}\right] \cos \left(\omega_{j} k \Delta t\right)-\operatorname{Im}\left[H_{j i m}\right] \sin \left(\omega_{j} k \Delta t\right) \ldots\right.} \\
& \left.-\operatorname{Im}\left[H_{j i m}\right] \cos \left(\omega_{j} k \Delta t\right)-\operatorname{Re}\left[H_{j i m}\right] \sin \left(\omega_{j} k \Delta t\right)\right]^{T}
\end{aligned}
$$

where $\Delta t$ denotes used sampling time. The full matrix at any point in time $t_{k}=k \Delta t$ is built by concatenating the submatrices $C_{j i m}$, leaving $k$ as the only free index (Pascoal and Soares, 2009).

The application of the Kalman filter (e.g. Brown and Hwang, 1992) involves the standard prediction and update cycles, and Pascoal and Soares (2009) carefully address many important aspects to consider when 
implementing the solution scheme in practice; including points about stabilisation of solution, conditioning of matrices, tuning of filter gain, etc.

It is important to mention that Pascoal and Soares (2009) consider station-kept ships, without forward speed, implying that the frequency $\omega$ in Eq. (9) is the (true) wave frequency. Thus, it is trivial to transform the wave spectrum, $S\left(\omega_{e}\right)$, estimated in encounter domain to $S(\omega)$ in the true domain. Although the extension to including forward speed is elementary, the practical incorporation is by no means straight-forward because of the Doppler shift (Eq. 2), leading to a 1-to-3 relationship between encounter and true frequency in following waves when $\omega_{e}<\frac{1}{4 A}$, cf. Eq. (2). The basic problem is identical to what is handled by the approach(es) formulated in the frequency domain (Section 3). However, it is not possible to use the same type of solutionscheme because of the different domains (frequency vs. time). In a new study by Pascoal et al. (2016), the effect of forward speed is reportedly included, but the article does not draft an actual implementation of it. On the other hand, one possible method to deal with the Doppler shift for ships having forward speed is suggested in a recent MSc study by Ding (2016), supervised by the present author. This MSc study shows how forward speed can be successfully included, so that the transformation of the wave spectrum from encounter to true domain is secured, but the implementation is restricted to long-crested waves. Hence, the extension to real ocean waves, i.e. full-scale experimental data, remains. The 'transformation problem' (for long-crested waves) is summarised in the following.

In case of nonzero forward speed, and for all waves approaching forward of 'beam sea', the transformation of the wave spectrum, $S\left(\omega_{e}\right)$, in the encounter domain to $S(\omega)$ in the true (frequency) domain is trivial, as Eq. (2) yields a 1-to-1 relationship. For waves approaching behind of 'beam sea' and $\omega_{e}<\frac{1}{4 A}$, the transformation is non-trivial, since Eq. (2) yields a 1-to-3 relationship; in case of a 1-to-1 relationship the transformation is straight-forward and identical to the former situation. If the 1-to-3 relationship occurs, the (suggested) solution is derived by considering the illustration shown in Figure 5. The estimated spectral ordinate $\hat{S}\left(\omega_{e}\right)=A_{e 1}$ in encounter-frequency domain is considered. Accordingly, the particular ordinate needs to be transferred into three ordinates in the (true) wave-frequency domain; $\hat{S}\left(\omega_{1}\right)=A_{\omega 1}$, $\hat{S}\left(\omega_{2}\right)=A_{\omega 2}$, and $\hat{S}\left(\omega_{3}\right)=A_{\omega 3}$, where (only) the frequencies $\left\{\omega_{1}, \omega_{2}, \omega_{3}\right\}$ are known, whereas the values $\left\{A_{\omega 1}, A_{\omega 2}, A_{\omega 3}\right\}$ are unknown. However, for any parameterised spectrum, $S^{*}\left(\omega \mid H_{s}, T_{z}, \ldots\right)$, known frequencies imply known spectral values, if the standard wave parameters $\left(H_{s}, T_{z}, \ldots\right)$ are also known. Thus, ratios between the spectral ordinates of the parameterised spectrum in the true domain and in the encounter domain, respectively, can be formed at the three known frequencies:

$$
\frac{S^{*}\left(\omega_{1}\right)}{S_{e}^{*}\left(\omega_{e}\right)}, \quad \frac{S^{*}\left(\omega_{2}\right)}{S_{e}^{*}\left(\omega_{e}\right)}, \quad \frac{S^{*}\left(\omega_{3}\right)}{S_{e}^{*}\left(\omega_{e}\right)}
$$




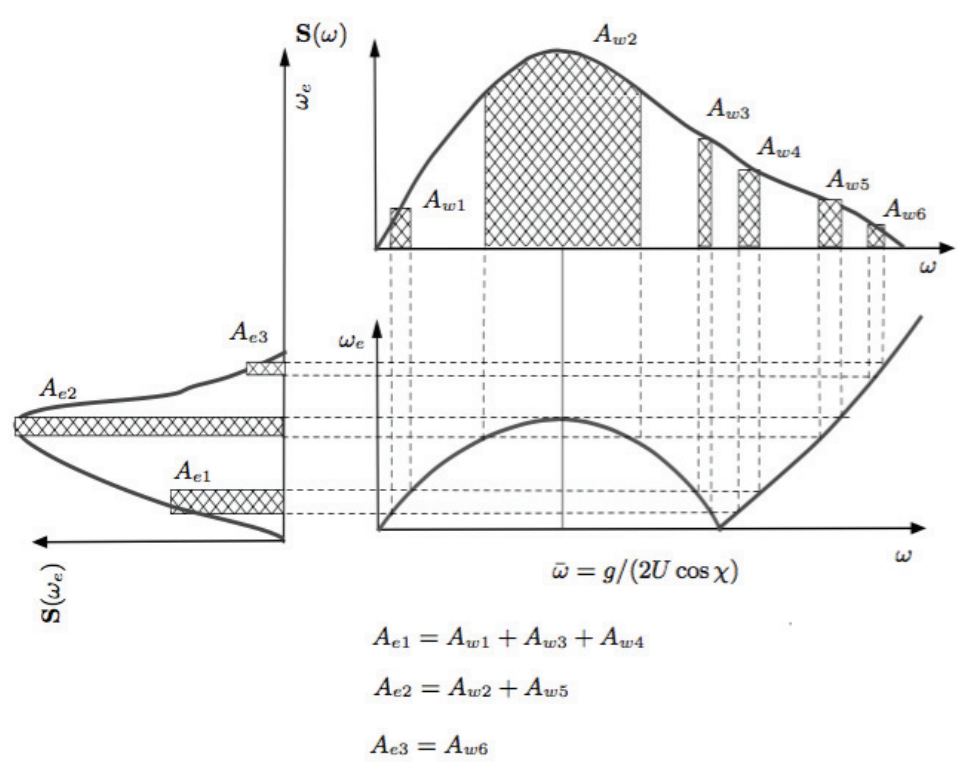

FIGURE 5. Transformation of wave spectrum. (Lewandowski, 2004)

taking note that $S_{e}^{*}\left(\omega_{e}\right)=S^{*}\left(\omega_{1}\right)+S^{*}\left(\omega_{2}\right)+S^{*}\left(\omega_{3}\right)$, cf. Figure 5. It may now be stated, or assumed, that the relative distribution of energy in the parameterised spectrum $S^{*}(\omega)$ reflects the distribution of the estimated "true" spectrum $\hat{S}(\omega)$. Hence, ratios similar to Eq. (11) can be formed from the estimated spectrum, and, relying on the stated assumption, the following expressions are derived:

$$
\begin{aligned}
& \frac{\hat{S}\left(\omega_{1}\right)}{\hat{S}\left(\omega_{e}\right)} \doteq \frac{S^{*}\left(\omega_{1}\right)}{S^{*}\left(\omega_{1}\right)+S^{*}\left(\omega_{2}\right)+S^{*}\left(\omega_{3}\right)} \\
& \frac{\hat{S}\left(\omega_{2}\right)}{\hat{S}\left(\omega_{e}\right)} \doteq \frac{S^{*}\left(\omega_{2}\right)}{S^{*}\left(\omega_{1}\right)+S^{*}\left(\omega_{2}\right)+S^{*}\left(\omega_{3}\right)} \\
& \frac{\hat{S}\left(\omega_{3}\right)}{\hat{S}\left(\omega_{e}\right)} \doteq \frac{S^{*}\left(\omega_{3}\right)}{S^{*}\left(\omega_{1}\right)+S^{*}\left(\omega_{2}\right)+S^{*}\left(\omega_{3}\right)}
\end{aligned}
$$

where the symbol ".” "expresses that the ratios are assumed to be identical; not necessarily with a match between the pairs of numerators and the pairs of denominators, respectively, on the left- and right-hand sides. In Eqs. (12)-(14), the estimated encounter wave spectral ordinate $\hat{S}\left(\omega_{e}\right)$ is formed by the complex wave amplitude, which is the output of the Kalman filtering approach. Thus, the estimated wave spectral ordinates $\hat{S}\left(\omega_{i}\right)$ at the three given wave frequencies $\omega_{i}, i=1,2,3$ can be calculated.

4.2. Wave Estimation Based on a Stepwise Procedure. New conceptual ideas for time-domain-based shipboard SSE were addressed recently (Bjerregård, 2014; Nielsen et al., 2015), and one particular method has been further studied by Nielsen et al. (2016). Stepwise, the method provides, first, the (characteristic) wave period obtained solely from a measured response signal. In the second step, the method combines the 
use of measurements and corresponding RAOs to estimate wave amplitude and phase (of a regular wave train). The partial independency of RAOs is representing a great advantage, as using them in real-world applications always is associated with uncertainty due to incomplete knowledge about the input conditions, i.e. speed, wave heading, draft, etc.; not to mention (in)accuracies in the calculation of the RAOs themselves.

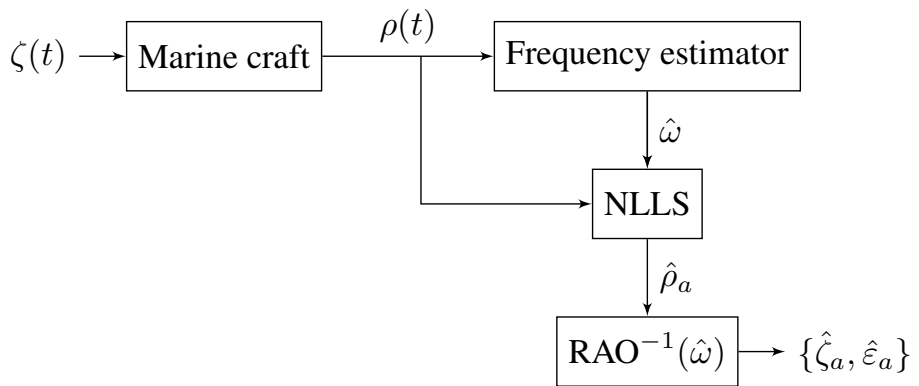

FIGURE 6. Estimation of wave elevation using nonlinear least squares fitting (NLLS) together with a 'frequency estimator'. (Nielsen et al., 2016)

The details of the stepwise procedure are left to Nielsen et al. (2016), but the principle of the procedure is summarised by the block diagram in Fig. 6. The "input" to the marine craft is a wave elevation signal $\zeta(t)$ and the "output", $\rho(t)$, is a corresponding motion response. From the motion response, the method provides, in the first step, the characteristic frequency $\hat{\omega}$ of the wave signal, and subsequently, in the second step, wave amplitude $\hat{\zeta}_{a}$ and phase $\hat{\varepsilon}_{a}$ are estimated, using a fitted value of the response amplitude $\hat{\rho}_{a}$ and the inverse, $\operatorname{RAO}_{\rho}^{-1}(\hat{\omega})$, of the corresponding transfer function. The 'Frequency estimator' is based on techniques developed within control theory (Aranovskiy et al., 2007; Belleter et al., 2015), whereas the estimated response amplitude $\hat{\rho}_{a}$ is found from a recursive nonlinear least squares (NLLS) optimisation (Nielsen et al., 2016). The estimation process is made on short sequences of data (5-7 wave periods), but a new estimation is made on shorter intervals, so that the analysis is a based on a "moving window" of data recordings, enabling real-time updates/estimates of the wave elevation. However, it is important to emphasise that the procedure is still far from mature to be applicable in sea state estimation from full-scale measurements data of ships, since the method, so far, is limited to handle only the estimation of regular wave trains based on corresponding response measurements from a ship without forward speed.

\section{ApPlicAtion STUDiES}

The previous sections have summarised different approaches for shipboard SSE, using the vessel itself as a wave buoy. In the past, the author has made numerous studies, alone and with colleagues, applying the aforementioned approaches both on simulated response data and on full-scale recordings for estimating the 
on-site sea state. However, the present section has not the aim to widely discuss or show the outcome of the studies and analyses; neither by words nor graphically. Instead, the purpose of this section is to point out some main findings from the mentioned studies dealing both with the frequency and the time domain approaches.

5.1. Frequency Domain SSE. Two main concepts for sea state estimation in the frequency domain were outlined in Section 3; (a) one based on a direct comparison of measured and theoretically calculated response spectra, and (b) another based on energy equivalence focusing on spectral moments. In case of the former concept (a), the sea state estimate is obtained by solving for each spectral ordinate of the wave spectrum; either by Bayesian modelling, strictly minimising a discrete version of Eq. (1), or by parametric modelling, optimising a set of sea state parameters of a given parameterised wave spectrum. In the concept based on energy equivalence (b), the sea state estimate is obtained by optimising also for a set of wave parameters, but considering the integrated variant of Eq. (1).

5.1.1. Direct Comparison of Spectral Energy Distribution. As outlined in Subsection 3.1, Bayesian or parametric modelling is applied to produce an estimate of the on-site sea state from comparison between spectra of corresponding responses. In either case, the final outcome is a frequency-directional wave spectrum as illustrated in Figure 7.
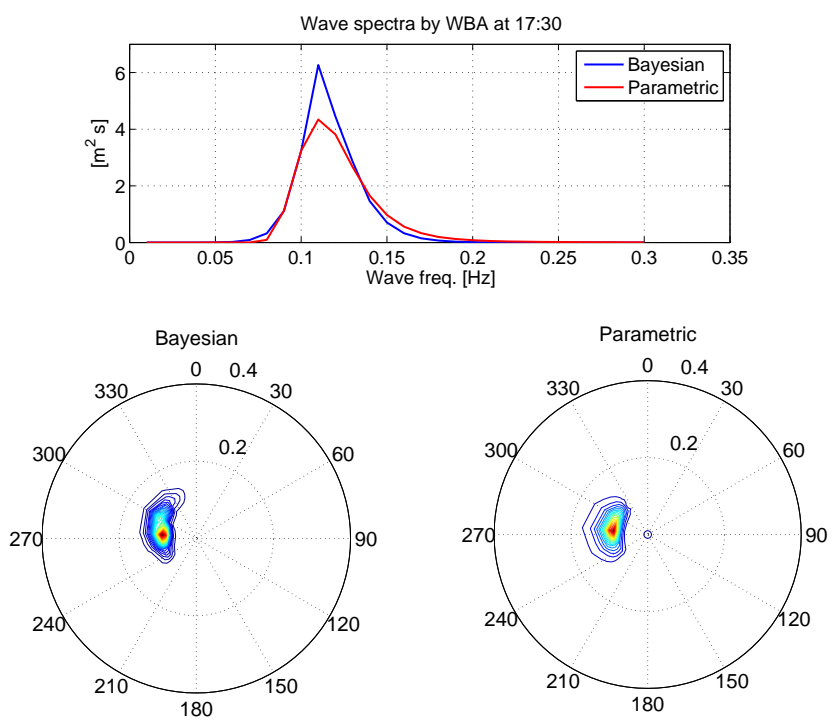

FIGURE 7. Typical wave spectrum obtained by wave buoy analogy; integrated frequency spectrum (top) and directional spectrum (bottom). Nielsen et al. (2013) 
It is the author's experience that the two techniques - Bayesian and parametric modelling - generally produce results with little deviation; in particular when integrated sea state parameters (significant wave height, peak period, mean wave direction, etc.) are considered. This has been confirmed in an extensive study by Nielsen et al. (2013), where more than 100 hours of response data from an in-service large container ship ( $L=349.0 \mathrm{~m}, B=42.8 \mathrm{~m}, T=14.5 \mathrm{~m}$ ) were analysed. Specifically, it was shown that daily statistics of integrated wave parameters agree well between the two sets of results. It is noteworthy that sea state estimates by other means, in this case wave radar data and hindcast studies, respectively, produced similar results. Figure 7 shows sample plots of the estimated wave spectrum corresponding to one particular instant in time (17:30 UTC, 12. Aug. 2011) based on 15 minutes of past measurements data; the results of Bayesian $(\mathrm{Hs}=2.2 \mathrm{~m})$ and parametric $(\mathrm{Hs}=2.0 \mathrm{~m})$ modelling are included. The whole data set, that is, all estimations are summarised in Figure 8, where the correlation between results of Bayesian and Parametric modelling, including wave radar data, is shown. Results are given for the significant wave height, the zero up-crossing period and the relative wave heading (180 deg. is head sea, positive values indicate waves from starboard side).
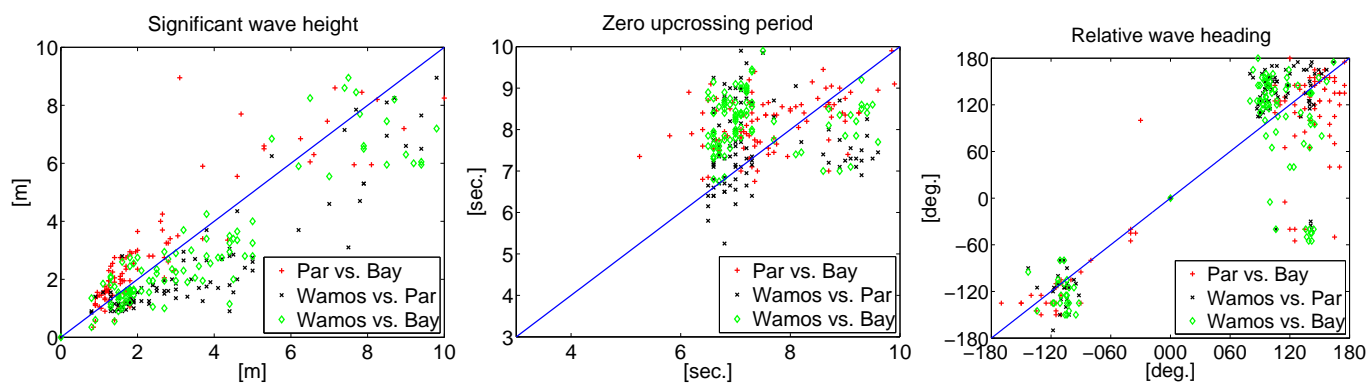

FIGURE 8. Correlations between estimates of integrated wave parameters as obtained by different shipboard techniques, including parametric (PAR) and Bayesian (Bay) modelling, respectively, and wave radar(Wamos).

In another comprehensive study by Nielsen and Stredulinksy (2012), focus was made on parametric modelling alone. In this study, sea trials motion data from a small research vessel $(L=71.5 \mathrm{~m}, B=12.8 \mathrm{~m}$, $T=4.8 \mathrm{~m}$ ) together with data from traditional wave buoys were analysed, and the purpose was to examine the sensitivity of sea state estimates by using sets of different vessel responses as input for the wave buoy analogy. The trials were carried out in the sea off Nova Scotia, Canada, with the detailed paths shown in Figure 9. Sample results are seen in Figure 10, where estimates by the wave buoy analogy (parametric modelling) are compared with measurements by a Triaxys ${ }^{T M}$ buoy. The plots are produced for one specific set of responses $\{$ roll angle, pitch angle, lateral acceleration $\}$ relative to the ship's COG, and it can be seen, that in these cases, the agreement is good; even for the multi-modal case shown at the right-hand side. 


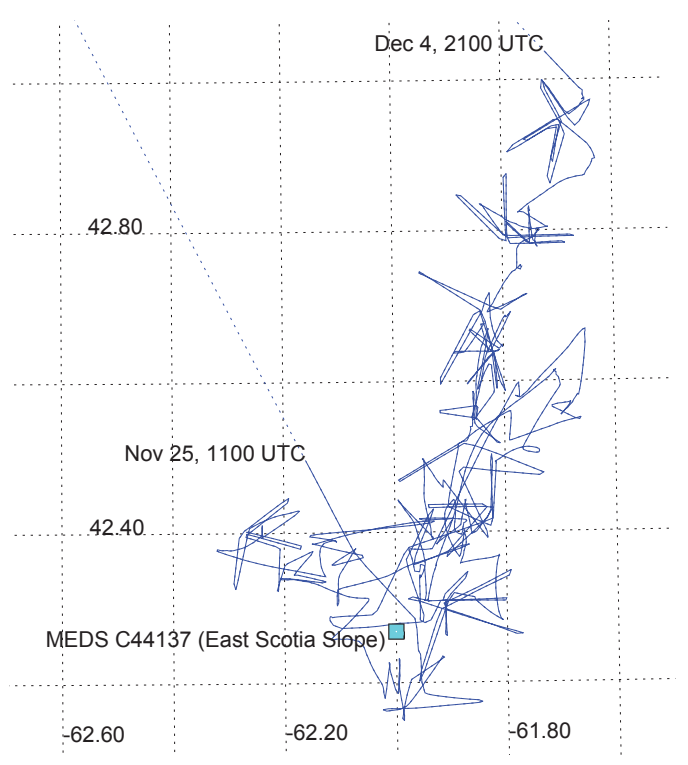

FIGURE 9. Voyage map, including detailed paths of individual trials.(Stredulinsky, 2010)

However, as pointed out in several publications (Tannuri et al., 2003; Pascoal et al., 2007; Simos et al., 2007;
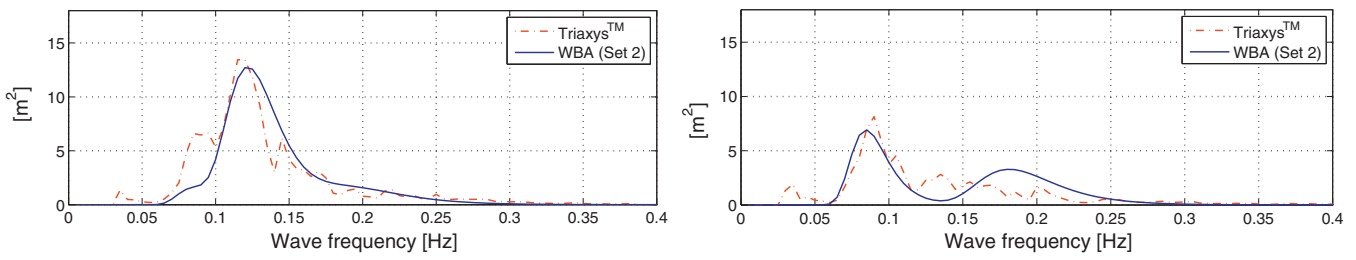

FIGURE 10. Estimated wave spectra (WBA) and 'measured' spectra (Triaxys). (Nielsen and Stredulinksy, 2012)

Sparano et al., 2008), and thoroughly analysed by Nielsen and Stredulinksy (2012), the selection of different combinations of motion components may significantly influence the sea state estimates from the wave buoy analogy. It is therefore of a particular concern to make sure that the most sensible set of motions/responses always is (automatically) selected. On the other hand, it is by no means straight-forward how to develop such a selection process, automatically and in real-time providing the best combination of responses; and work in this area is still ongoing with auspicious results in a newly publication by Montazeri et al. (2016b) that introduce an approach based on local sensitivity analysis.

In terms of accuracy and general results in previous studies, it is difficult for the author to favour the one modelling procedure to the other, and the computational efficiency of the methods is also comparable. Typically, an estimate is provided in about 5 minutes on a standard PC (Intel(R) Core(TM) i7-4600U @ 2.10 $\mathrm{GHz}$ ). In practice, this means that "real-time" updates of sea states are possible, as a sea state, for most 
purposes, is taken to be stationary for periods of approximately 20 minutes; only accounting for the sea state itself and not any change in vessel speed and/or heading which would lead to nonstationary responses.

The most notable difference between the two procedures is probably the ability of the Bayesian method to (better) estimate wave spectra which do not follow "standard parametric shapes", since each spectral component of the wave spectrum is solved for (i.e. estimated) individually. On the other hand, for most practical cases, a summation of parameterised wave spectra like, for instance, JONSWAP can be fitted to represent most ocean wave spectra, like it was seen in Figure 10 for the multi-modal case on the right-hand side.

5.1.2. Energy Equivalence: Comparison of Spectral Moments. In the recently developed method, based on energy equivalence, the ambition by the authors (Montazeri et al., 2016a) was partly to make a practical robust procedure and partly to increase the computational efficiency of the wave buoy analogy; without affecting the ability to provide accurate sea state estimates. This ambition is strived for by optimising the wave parameters of a parameterised spectrum, containing a swell system and a wind sea system, using a partitioning technique to estimate separately the individual systems. Clearly, due to its recent development, the method needs to be further tested but, based on preliminary analyses of simulated data, promising results have been obtained. The performance of the method has been examined thoroughly by Montazeri et al. (2016a), testing the method's capability to estimate both unimodal and bimodal wave spectra, generated by pure wind sea and, respectively, mixed sea (wind + swell) conditions. In the study, simulations of motion responses were carried out for a container ship ( $L=349.0 \mathrm{~m}, B=42.8 \mathrm{~m}, T=14.5 \mathrm{~m}$ ) similar to that studied in the previous subsection. Sample results of the study can be seen from Table 1, which presents the outcome of four test cases, $\mathrm{M}$ to $\mathrm{P}$, all representing a mixed sea condition, with a wind sea and a swell part. From the table, the true wave parameters for both parts appear; $H_{s}$ is significant wave height, $T_{p}$ is peak period of the particular spectrum (wind sea or swell), $\mu$ is relative wave heading, $s_{\text {max }}$ is maximum spreading parameter (Montazeri et al., 2016a). In the analysis, the true parameters have been used to simulate 15 stochastic wave realisations, including corresponding sets of vessel responses, for each case. Subsequently, one set of vessel responses at a time has been used as input for the estimation method, and the outcome is a set of corresponding estimated wave parameters. Thus, mean values and associated standard deviations were obtained for the 15 realisations of each case $(\mathrm{M}, \mathrm{N}, \mathrm{O}, \mathrm{P})$. It is noteworthy that the sensitivity to the used set of transfer functions, i.e. RAOs, was investigated by using RAOs calculated by two different sets of software, say, I and II; but except from that the RAOs have been computed for the same responses under the exact same input conditions with respect to draft, speed, etc. In the one situation, labelled 'RAO1', the RAOs of software I were used to both simulate the stochastic wave realisations and to subsequently estimate the sea 
TABLE 1. True and estimated wave parameters obtained in a comprehensive simulation

study focusing on SSE based on energy equivalence. (Montazeri et al., 2016a)

\begin{tabular}{|c|c|c|c|c|c|c|c|c|c|}
\hline \multirow[t]{2}{*}{ Case } & & \multicolumn{4}{|c|}{ Wind sea } & \multicolumn{4}{|l|}{ Swell } \\
\hline & & $H_{s}(\mathrm{~m})$ & $T_{p}(\mathrm{~s})$ & $\mu$ (deg.) & $s_{\max }$ & $H_{s}(\mathrm{~m})$ & $T_{p}(\mathrm{~s})$ & $\mu$ (deg.) & $S_{\max }$ \\
\hline \multirow[t]{5}{*}{ I } & True & 3 & 8 & 45 & 10 & 5 & 15 & -135 & 25 \\
\hline & mean (RAO1) & 3.1 & 8.8 & 66 & 15 & 5.2 & 15 & -160 & 33 \\
\hline & mean (RAO2) & 4 & 8.5 & 52 & 15 & 4.2 & 15 & -101 & 60 \\
\hline & std (RA01) & 0.7 & 0.49 & 10 & 0 & 0.6 & 0.5 & 12 & 5.8 \\
\hline & std (RAO2) & 0.3 & 0.55 & 1.5 & 5 & 0.8 & 0.4 & 13 & 20 \\
\hline \multirow[t]{5}{*}{ J } & True & 3 & 8 & -90 & 10 & 5 & 15 & 90 & 25 \\
\hline & mean (RAO1) & 3.2 & 8.6 & -106 & 18 & 4.4 & 16.6 & 98 & 27 \\
\hline & mean (RAO2) & 3.6 & 9.2 & -92 & 20 & 3.8 & 15.1 & 120 & 47 \\
\hline & std (RAO1) & 0.57 & 0.75 & 22 & 2 & 1.3 & 1 & 7.6 & 14 \\
\hline & std (RAO2) & 0.25 & 1.3 & 17 & 0 & 0.08 & 0.6 & 30 & 20 \\
\hline \multirow[t]{5}{*}{ K } & True & 3 & 8 & 135 & 10 & 5 & 15 & 45 & 25 \\
\hline & mean (RAO1) & 2.3 & 7.3 & 120 & 12 & 5.5 & 13 & 49 & 65 \\
\hline & mean (RAO2) & 3.4 & 6.7 & 141 & 15 & 5.8 & 12 & 3 & 64 \\
\hline & std (RAO1) & 0.5 & 0.8 & 13 & 0 & 0.4 & 0.1 & 14 & 20 \\
\hline & std (RAO2) & 0.2 & 0.2 & 10 & 3 & 0.5 & 0.1 & 16 & 23 \\
\hline \multirow[t]{5}{*}{$\mathrm{L}$} & True & 3 & 8 & 90 & 10 & 5 & 15 & 180 & 25 \\
\hline & mean (RAO1) & 3.6 & 9.1 & 89 & 15 & 5.3 & 16 & 174 & 49 \\
\hline & mean (RAO2) & 3.3 & 6.8 & 100 & 18 & 5.8 & 14 & 176 & 59 \\
\hline & std (RAO1) & 0.5 & 0.1 & 2 & 0 & 0.9 & 2.7 & 4 & 28 \\
\hline & std (RAO2) & 1 & 0.9 & 2 & 4 & 0.8 & 0.6 & 11 & 12 \\
\hline
\end{tabular}

state. In the other situation, labelled 'RAO2', RAOs of software I were used for the simulation part, whereas RAOs of software II were used in the estimation part. This latter situation resembles a situation close(r) to reality, since the 'input conditions' are never exactly known during full-scale operational service. On average, the best estimates are observed for 'RAO1'; both in terms of mean values and standard deviations. However, the important point in this context is that reasonable estimates are found even for 'RAO2'; and the differences between the results of 'RAO1' and 'RAO2' are barely noticeable from Table 1. Altogether, good agreement is found between the true and the estimated wave parameters and the study proves both robustness and computational efficiency of the proposed method. A number of other interesting findings are reported by the original paper (Montazeri et al., 2016a), but no further remarks are given in the present review.

5.2. Time domain SSE. The number of studies focusing on the time domain procedures, Kalman filtering and the stepwise procedure, respectively, are (still) limited compared to the number of frequency-domain studies. In the future, this is likely to change and the present subsection briefly summarises some of the existing work that further elaborations may rely on.

5.2.1. Kalman Filtering. In the first study (Pascoal and Soares, 2009), where Kalman filtering was applied, the implementation was made for the zero-forward speed case only, and just numerical simulations of motion data were studied. Similar to any of the frequency domain procedure, the introduction of advance speed in 
the Kalman filtering approach is elementary, in theory; however, in practice, the implementation is by no means straight-forward. The study by Pascoal et al. (2016) addresses partly the effect of forward speed, but only in a "qualitative manner", since the work does not describe any details about the actual implementation of advance speed. On the other hand, the practical implementation of the advance-speed problem was the very topic of a recent MSc thesis by Ding (2016), supervised by the present author. The MSc study shows how forward speed can be successfully included, but the implementation is restricted to long-crested waves, and, as a consequence, simulation data is studied only for which reason there is still further work to be made. One test case from the study is shown in Figure 11, which applies for a container vessel ( $L=175.0 \mathrm{~m}$, $B=25.4 \mathrm{~m}, T=9.5 \mathrm{~m}$ ) at speed 10 knots in stern quartering waves. The plot shows the statistics, i.e. average, of totally fifty estimations with the same true input wave parameters: $H_{s}=4.0 \mathrm{~m}, T_{z}=10.0 \mathrm{~s}$, $\chi=045 \mathrm{deg}$. The individual estimation is based on a set of simulations of two responses, heave and pitch, realised from a wave elevation process with the given (true) wave parameters. As can be seen from the plot, the agreement between the estimated spectrum and the true spectrum is, on average, good; both in terms of the total area under the spectra (= energy of the wave system), measured by the significant wave height, and the location of the spectra' peak, taken as the peak frequency. However, while the estimated peak frequency consistently agrees well with the true peak frequency, some variation in the amount of energy is observed; seen from the dashed lines representing the lower and upper extremes of the estimated spectra considering all fifty outcomes. Evaluated by numbers, the mean significant wave height is $\hat{H}_{s}=3.7 \mathrm{~m}$ with the coefficient of variation being 0.17 .

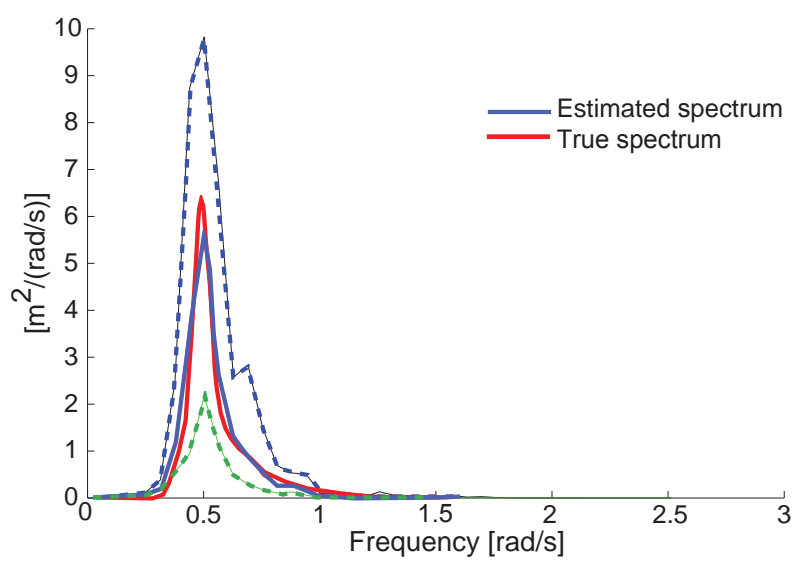

FIGURE 11. Wave estimation using Kalman filtering. Full lines indicate 'average spectrum', while dashed lines represent lowest and highest energy content in estimated spectrum, obtained from fifty sets of estimations. (Ding, 2016) 
5.2.2. Stepwise Procedure. The stepwise method is limited to handle only the estimation of regular wave trains from corresponding response measurements on a ship without forward speed. However, very promising results have been found in simulation studies and from model-scale experiments outlined by Nielsen et al. (2015) and Nielsen et al. (2016), respectively. In the former studies, one of the test cases focuses on a container $\operatorname{ship}(L=349.0 \mathrm{~m}, B=42.8 \mathrm{~m}, T=14.5 \mathrm{~m}$ ) being exposed to a regular wave train described by a wave frequency $\omega=0.6 \mathrm{rad} / \mathrm{s}$ and an amplitude $\zeta_{a}$. The value of the amplitude is initially $1.0 \mathrm{~m}$ but increases to $\zeta_{a}=2.0 \mathrm{~m}$ during a short period of time [300;320] $\mathrm{s}$. The simulation of the wave train includes measurement noise, taken as Gaussian white noise produced with a $12 \mathrm{~dB}$ SNR and, with this "seaway" as input, the heave response is simulated in bow-quartering long-crested waves (relative wave heading equal to $135 \mathrm{deg}$ ). The wave amplitude estimate, from one simulation, is shown in Figure 12a, where the stairs are explained because estimation is made on short sequences of data (5-7 wave periods), cf. subsection 4.2. The complete reconstruction of the wave elevation process can be seen in Figure 12b. The plots show that the wave parameters, including the actual time history, are estimated with good accuracy. The reason to test on a case with a somewhat nonphysical sudden change in wave amplitude is merely to evaluate the estimation method's ability to handle nonstationary data; one of the most important capabilities of the method, since the method was developed to possess this very property. Indeed, a good result is achieved, and other similar test cases, but with an abrupt change in wave frequency instead, show equally good behaviour.

The stepwise method has also been tested with experimental data (Nielsen et al., 2016), where a 1:30 scalemodel of a platform supply vessel has been exposed to regular waves in the model-basin at the Marine Cybernetics Laboratory (MCLab) at NTNU, Trondheim, cf. Figure 13.

A number of test cases were considered in the experiments and one is presented in Figures 14 and 15. Specifically, this test case involves wave estimation based on measurements of the heave response in beam sea condition, see Figure 14, where wave amplitude and period were fixed at $\{2.0 \mathrm{~cm}, 1.2 \mathrm{~s}\}$ and $\{3.0 \mathrm{~cm}, 1.7 \mathrm{~s}\}$, denoted by Case D and Case E, respectively. In both situations, the actual wave train has been estimated from 200 seconds data recordings and the results are seen in Figure 15, where the individual plot represents a zoom of a time window selected arbitrarily from the full estimation sequence lasting 200 seconds in both cases. In the plots the true amplitude levels are indicated, and it is seen that the estimated results are good. For the two cases, the estimated (mean) wave periods are $T_{\text {est }}=1.21 \mathrm{~s}$ and $T_{\text {est }}=1.71 \mathrm{~s}$, which agree nicely with the true periods. In the model-basin, the wave elevation is usually measured by a wave probe but, unfortunately, the probe was malfunctioning on the days when the experiments were conducted. Consequently, no comparisons can be made between the estimated wave elevation and the actual true one. As another remark, it should be noted that the "simulations-only" examples, addressed by Nielsen et al. (2015), 


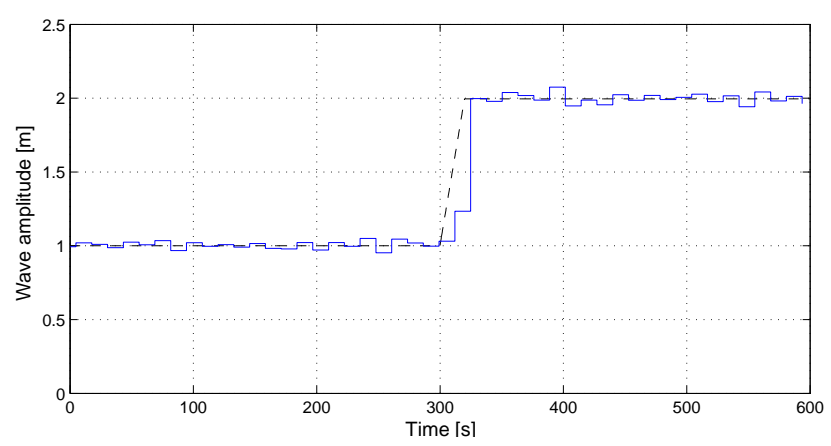

(a) Estimated wave amplitude (full line) and true one (dashed line).

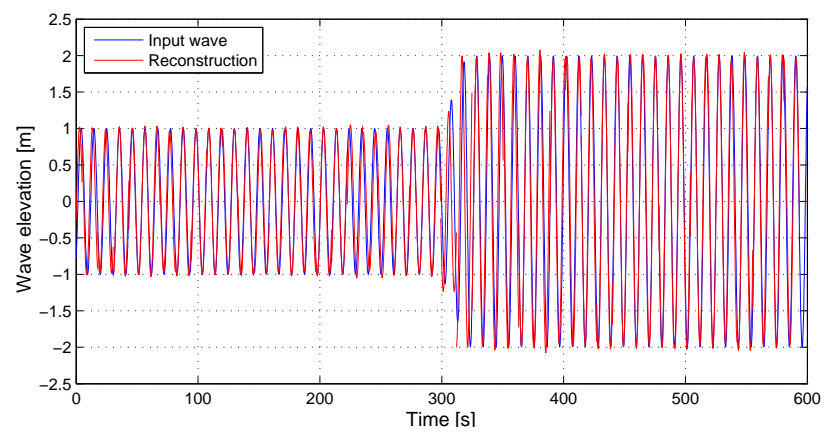

(b) Reconstruction of wave elevation process.

FIGURE 12. Sea state estimation based on heave response of a container vessel exposed to bow-quartering regular waves ( $U=0$ knots), including measurement noise (12 dB SNR).

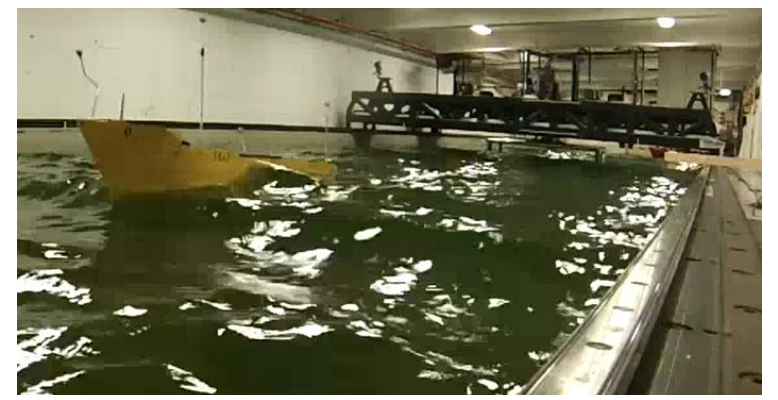

FIGURE 13. Experimental facilities at the Marine Cybernetics Laboratory, NTNU.

(Brodtkorb et al., 2015)

were studying also the capability to handle nonstationary conditions, such as sudden changes to the (true) wave parameters of the wave train to be estimated. The same kind of experiments cannot not be made in the model-testing facility, since it is possible only to change the control mechanism of the wave generator after a full stop. 


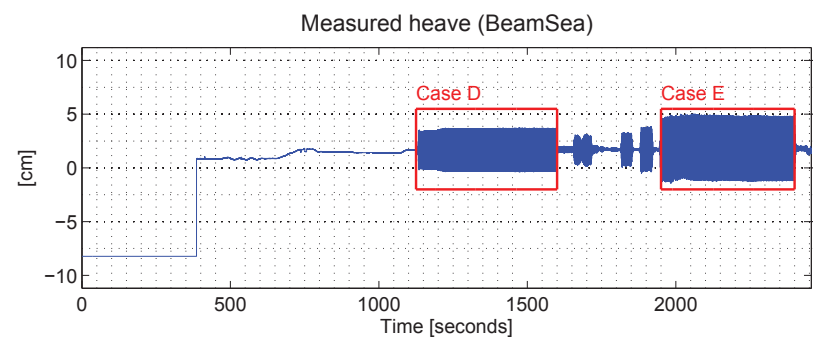

FIGURE 14. Samples of time history recordings of the response measurements. Cases $D$ and $E$ represents the heave motion. In the post-analysis, the measurements have been averaged to zero-mean. (Nielsen et al., 2016)
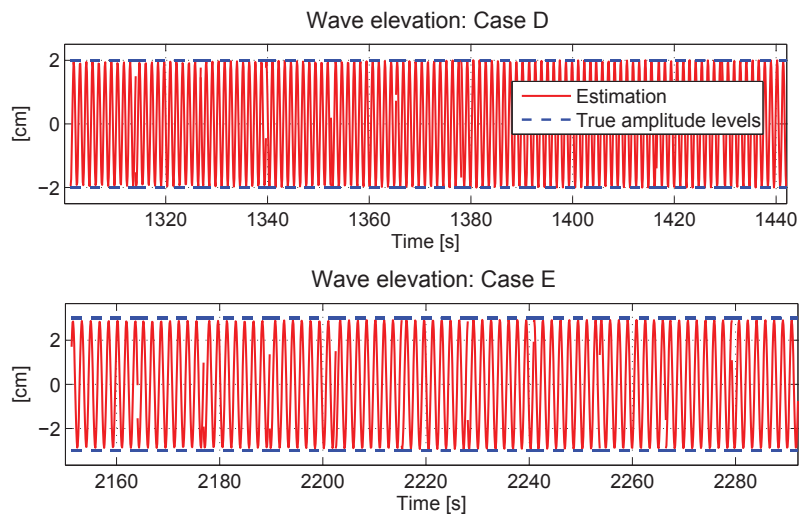

FIGURE 15. Estimation of wave elevation history from model-scale experiments. (Nielsen et al., 2016)

Obviously, the stepwise estimation method must be extended before it has practical relevance as a means for shipboard SSE along with the other techniques addressed in the present paper. Two notable points in future suggested studies are: (1) The extension to consider regular wave trains composed by two wave components could be beneficial, as it would provide knowledge about how to handle estimation of an irregular wave train made up by a (very) large number of regular wave components. Specifically, work could address the use of several notch or bandpass filters to select individual harmonic components from a wave spectrum, and then use a 'regular wave estimator', like the developed one, for each component. (2) The combination/consideration of several responses simultaneously, e.g., $\{$ heave; roll; pitch $\}$ could (possibly) be used to estimate also the relative wave heading. 


\section{CONCLUding REMARKS}

Procedures for shipboard sea state estimation based on measured vessel responses have been studied and developed since the 1970'ies. The concept of the wave buoy analogy is still not widely used in practice, but it is the author's opinion that generally it has matured to a level that would be applicable for shipboard decision support systems. The concept offers a reasonable alternative to the other shipboard estimating means, i.e. wave radars, but the concept has lower costs and requires no (or very little) calibration compared to wave radars. On the other hand, the wave buoy analogy still has weak points which need to be further addressed. Notably, the ability to handle nonstationary data, which may compromise accuracy/reliability of real-time sea state estimates. Moreover, it would be beneficial to be able to automatically select the best combination of available vessel responses; taking into account the effect of different operational conditions. In the same context, clearly there is a need to introduce fault detection and fault tolerance since, by nature, all sensor signals will be faulty at times. Obviously, and beyond doubt, this point is of importance to not only the wave buoy analogy, but to all components of shipboard monitoring and decision support systems. It is also important to point out, when using a ship as a wave buoy, the inherent limited capability to estimate waves not necessarily felt by the ship, because of the ship's motion characteristics making it a wave filter. This issue is touched in a number of previous work (Nielsen, 2006, 2007; Pascoal and Soares, 2008) and a means to mitigate partly the problem is to use a response such as the relative wave height (Nielsen, 2008a); based on the instantaneous distance from a fixed point at/on the hull to the sea surface, measured e.g. by pressure transducers (below the water line) and/or distance meters mounted on the railing. In the same line, it could be interesting to consider sensor fusion, since generally wave radars are considered to yield accurate estimates of wave period and direction but not always wave height; leaving out any other pro/cons related to the use of wave radars. In case of sensor fusion, the Kalman filter approach offers a good setting (Pascoal and Soares, 2009). Finally, efforts should look into associating uncertainty measures on the particular wave parameter estimates by the wave buoy analogy.

The immediate use of sea state estimates onboard a ship is directly coupled to navigational guidance and decision support to the ship's master and crew, where focus is on safety and fuel consumption. In a somewhat bigger perspective other uses of shipboard sea state estimations are, for instance, linked to:

- Ships' operational profiles in a short-term sense and during their lifetime; where an issue is whether a ship meets the wave scenarios as it was designed for, notably with respect to safety and speed.

- On-shore performance evaluation of a ship and entire fleets; shipping companies should be able to make more qualified fuel performance evaluations and comparisons when (reliable) wave data at actual position(s) is available. 
- Added resistance in waves; related to the previous point, it is desirable to have knowledge about and to improve models for calculating the added resistance in waves, where experimental data is still scarce.

- Global network of 'wave recorders'; the total number of ships navigating the oceans is very large and, if connected in a network, an enormous amount of wave data/statistics becomes available.

- Investigation of accidents; a sort of 'black box' could be installed on ships like it is known from the aviation industry. Thus, with information about responses as well as wave conditions, weather- and wave-induced accidents would be easier to investigate and analyse.

\section{ACKNOWLEDGEMENT}

The author is pleased to acknowledge his long time collaboration with Professors Jørgen Juncher Jensen, Technical University of Denmark, and Toshio Iseki, Tokyo University of Marine Science and Technology; who were the persons to introduce the author to the topic of shipboard sea state estimation. Additionally, and as a "post-process", the manuscript was improved by the assistance of anonymous reviewers.

\section{REFERENCES}

S. V. Aranovskiy, A. A. Bobtsov, A. S. Kremlev, and G. V. Lukyanova. A robust algorithm for identification of the frequency of a sinusoidal signal. Journal of Computer and Systems Sciences International, 46: 371-376, 2007.

D. J. W. Belleter, R. Galeazzi, and T. I. Fossen. Experimental verification of a global exponential stable nonlinear wave encounter frequency estimator. Ocean Engineering, 97:48-56, 2015.

M. Bjerregård. Methods for sea state estimation. Master’s thesis, Technical University of Denmark, Kgs. Lyngby, Denmark, June 2014.

A. V. Boukhanovsky and C. Guedes Soares. Modelling of multipeaked directional wave spectra. Applied Ocean Research, 31:132-141, 2009.

A. H. Brodtkorb, U. D. Nielsen, and A. J. Sørensen. Sea State Estimation Using Model-scale DP Measurements. In Proc. of MTS/IEEE OCEANS15, Washington, DC, USA, 2015.

R. G. Brown and P. Y. C. Hwang. Introduction to Random Signals and Applied Kalman Filtering. John Wiley \& Sons, Inc., 3rd edition, 1992.

F.W. Debord and B. Hennessy. Development of a Generalized Onboard Response Monitoring System. Technical report, Ship Structure Committee, 1990. Report SSC-349.

L. Ding. Kalman filtering applied to wave spectrum estimation based on measured vessel responses. Master's thesis, Technical University of Denmark, Kgs. Lyngby, Denmark, June 2016. 
A. Francescutto. Is It Really Impossible to Design Safe Ships? Trans. RINA, 135:163-168, 1993.

T. Hirayama. Real-time Estimation of Sea Spectra Based on Motions of a Running Ship. J Kansai Soc Naval Arch, 204:21-27, 1987.

J. Hua and M. Palmquist. Wave Estimation through Ship Motion Measurement. Technical report, Naval Architecture, Department of Vehicle Engineering, Royal Institute of Technology, 1994.

T. Iseki. Extended Bayesian Estimation of Directional Wave Spectra. In Proceedings of 23rd Int. Conf. on Offshore Mechanics and Arctic Engineering (OMAE), Vancouver, Canada, 2004. ASME.

T. Iseki and U. D. Nielsen. Study on a Short-term Variability of Ship Responses in Waves. Journal of Japan Institute of Navigation, 132:51-57, 2015.

T. Iseki and K. Ohtsu. Bayesian estimation of directional wave spectra based on ship motions. Control Engineering Practice, 8:215-219, 2000.

T. Iseki and D. Terada. Bayesian Estimation of Directional Wave Spectra for Ship Guidance Systems. International Journal of Offshore and Polar Engineering, 12:25-30, 2002.

T. Iseki, K. Ohtsu, and M. Fujino. A Study on Estimation of Directional Spectra Based on Ship Motions. Journal of Japan Institute of Navigation, 86:179-188, 1992.

M. Isobe, K. Kondo, and K. Horikawa. Extension of MLM for Estimating Directional Wave Spectrum. In Proceedings of Symposium on Description and Modeling of Directional Seas, volume A-6, 1984.

K. Kobune and N. Hashimoto. Estimation of Directional Spectra from the Maximum Entropy Principle. In Proceedings of 5th Int. Offshore Mechanics and Arctic Engineering (OMAE) Symposium, pages 80-85, Tokyo, Japan, 1986. ASME.

E. M. Lewandowski. The Dynamics of Marine Craft: Maneuvering and Seakeeping. World Scientific, 2004. K. Lindemann and N. Nordenstrøm. A System for Ship Handling in Rough Weather. In Proc. of Fourth Ship Control System Symposium, volume 7, pages 45-68, Royal Netherlands Naval College, 1975.

K. Lindemann, J. Odland, and J. Strengehagen. On the Application of Hull Surveillance Systems for Increased Safety and Improved Structural Utilization in Rough Weather. Trans. of SNAME, 85:131-166, 1977.

K. Maeda, T. Akashi, and K. Saito. An estimation of ocean wave characteristics based on measured ship motions (4th report): Directional wave spectrum estimated from full-scale measurements. J. of the Society of Naval Architects of Japan, 190:241-246, 2001.

C. Møgster. Bayesian methods for estimating non-stationary ship response spectra. Master's thesis, Norwegian University of Science and Technology, Trondheim, Norway, May 2015.

N. Montazeri. Estimation of waves and ship responses using onboard measurements. $\mathrm{PhD}$ thesis, Section of Fluid Mechanics, Coastal and Maritime Engineering, Department of Mechanical Engineering, Technical 
University of Denmark, June 2016.

N. Montazeri, U. D. Nielsen, and J. J. Jensen. Estimation of wind sea and swell using shipboard measurements - A refined parametric modelling approach. Applied Ocean Research, 54:73-86, 2016a.

N. Montazeri, U. D. Nielsen, and J. J. Jensen. Selection of the optimum combination of responses for Wave Buoy Analogy - An approach based on local sensitivity analysis. In Proc. of 13th PRADS, Copenhagen, Denmark, 2016b.

U. D. Nielsen. Estimation of Directional Wave Spectra from Measured Ship Responses. PhD thesis, Section of Coastal, Maritime and Structural Engineering, Department of Mechanical Engineering, Technical University of Denmark, May 2005.

U. D. Nielsen. Estimations of on-site directional wave spectra from measured ship responses. Marine Structures, 19:33-69, 2006.

U. D. Nielsen. Response-based estimation of sea state parameters - influence of filtering. Ocean Engineering, 34:1797-1810, 2007.

U. D. Nielsen. The wave buoy analogy - estimating high-frequency wave excitations. Applied Ocean Research, 30:100-106, 2008a.

U. D. Nielsen. Introducing two hyperparameters in Bayesian estimation of wave spectra. Probabilistic Engineering Mechanics, 23:84-94, 2008b.

U. D. Nielsen and T. Iseki. A Study on Parametric Wave Estimation Based on Measured Ship Motions. Journal of Japan Institute of Navigation, 126, 2012.

U. D. Nielsen and T. Iseki. Prediction of First-Order Vessel Responses with Applications to Decision Support Systems. In Proc. of 5th World Maritime Technology Conference, Providence, RI, USA, 2015.

U. D. Nielsen and D. C. Stredulinksy. Sea state estimation from an advancing ship - A comparative study using sea trial data. Applied Ocean Research, 34:33-44, 2012.

U. D. Nielsen, I. M. V. Andersen, and J. Koning. Comparisons of Means for Estimating Sea States from an Advancing Large Container Ship. In Proc. of 12th PRADS, Changwon, South Korea, 2013.

U. D. Nielsen, M. Bjerregård, R. Galeazzi, and T. I. Fossen. New Concepts of Shipboard Sea State Estimation. In Proc. of MTS/IEEE OCEANS15, Washington, DC, USA, 2015.

U. D. Nielsen, R. Galeazzi, and A. H. Brodtkorb. Evaluation of Shipboard Wave Estimation Techniques Through Model-scale Experiments. In Proc. of MTS/IEEE OCEANS16, Shanghai, China, 2016.

M. K. Ochi. Applied probability and stochastic processes in engineering and physical sciences. Wiley, 1990.

R. Pascoal and C. Guedes Soares. Non-parametric wave spectral estimation using vessel motions. Applied Ocean Research, 30:46-53, 2008. 
R. Pascoal and C. Guedes Soares. Kalman filtering of vessel motions for ocean wave directional spectrum estimation. Ocean Engineering, 36:477-488, 2009.

R. Pascoal, L. P. Perera, and C. Guedes Soares. Estimation of Directional Sea Spectra from Ship Motions in Sea Trials. To appear in Ocean Engineering, 2016.

R. Pascoal, C. Guedes Soares, and A. J. Sørensen. Ocean Wave Spectral Estimation Using Vessel Wave Frequency Motions. Journal of Offshore Mechanics and Arctic Engineering, 129:90-96, 2007.

J.A. Pinkster. Wave-feed-forward as a means to improve dynamic positioning. In Proceedings of Offshore Technology Conference (OTC), Houston, USA, 1978.

D.W. Robinson. Voyage Data Recorders - Operating with Safety and Efficiency. In Proceedings of IMAS90: Marine Technology and the Environment, London, Institute of Marine Engineers, May 1990.

K. Saito, K. Maeda, A. Matsuda, and K. Suzuki. An Estimation of Wave Characteristics Based on Measured Ship Motions (3rd Report). J. of the Society of Naval Architects of Japan, 187:77-83, 2000.

A. N. Simos, J. V. Sparano, E. A. Tannuri, and V. L. F. Matos. Directional Wave Spectrum Estimation Based on a Vessel 1st Order Motions: Field Results. In Proc. of 17th International Offshore and Polar Engineering Conference, Lisbon, Portugal, 2007.

J. V. Sparano, E. A. Tannuri, A. N. Simos, and V. L. F. Matos. On the Estimation of Directional Wave Spectrum Based on Stationary vessels 1st Order Motions: A New Set of Experimental Results. In Proc. of OMAE'08, Lisbon, Portugal, 2008.

M. St.Denis and W.J. Pierson. On the Motion of Ships in Confused Seas. Trans. of SNAME, 61:280-332, 1953.

D. C. Stredulinsky. Quest Q319 Sea Trial Summary and Wave Fusion Analysis. Technical Report TM 2010051, Defence Research and Development (DRDC) Canada - Atlantic, Dartmouth, NS, Canada, 2010.

K. Takekuma and T. Takahashi. On the Evaluation of Sea Spectra based on the Measured Ship Motions. Trans. of the West-Japan Society of Naval Architects, 45:51-57, 1973.

E. A. Tannuri, J. V. Sparano, A. N. Simos, and J. J. Da Cruz. Estimating directional wave spectrum based on stationary ship motion measurements. Applied Ocean Research, 25:243-261, 2003.

O.J. Waals, A.B. Aalbers, and J.A. Pinkster. Maximum Likelihood Method as a Means to Estimate The Directional Wave Spectrum and The Mean Wave Drift Force on a Dynamically Positioned Vessel. In Proceedings of OMAE2002, Oslo, Norway, 2002. ASME. 